\title{
Influence of cation vacancy related defects on the self- assembly processes in CdSe/ZnSe quantum dot heterostructures
}

\author{
L.V. Borkovska ${ }^{1}$, N.O. Korsunska ${ }^{1}$, V.I. Kushnirenko' ${ }^{1}$, Yu.G. Sadofyev², M.K. Sheinkman' \\ ${ }^{1} \mathrm{~V}$. Lashkaryov Institute of Semiconductor Physics, NAS of Ukraine, 45 prospect Nauky, 03028 Kyiv, Ukraine \\ Phone: +38(044) 265 7234; fax: +38(044) 265 8342; e-mail: bork@lumin.semicond.kiev.ua \\ ${ }^{2}$ Lebedev Physical Institute RAS, 53 Leninskii pr., 117924 Moscow, Russia
}

\begin{abstract}
A spatial distribution of the cation vacancy related defects and their influence on the formation processes of self-assembled nanoislands in CdSe/ZnSe heterostructures were investigated by photoluminescence methods. Self-assembling growth was achieved under low temperature $\left(230^{\circ} \mathrm{C}\right)$ molecular beam epitaxy with a subsequent annealing step. To change a number of cation vacancy related defects a VI/II beam pressure ratio $\mathrm{P}_{\mathrm{VI}} / \mathrm{P}_{\mathrm{II}}$ was varied from $2: 1$ to $5: 1$. In the samples grown under $\mathrm{P}_{\mathrm{VI}} / \mathrm{P}_{\mathrm{II}}=5: 1$ a significant increase of self activated emission band caused by cation vacancy related defects was found. A study of the excitation spectra of defect related band revealed that in all samples the cation vacancy related defects are present in $\mathrm{ZnCdSe}$ wetting layer. In the samples grown under $\mathrm{P}_{\mathrm{VI}} / \mathrm{P}_{\mathrm{II}}=5: 1$ they were observed on nanoisland interface too. It was found that the increase of Se beam pressure results also in high energy shift and narrowing of nanoisland emission band. This process is accompanied by low energy shift of $\mathrm{ZnSe}$ band-to-band emission. Observed changes in photoluminescence spectra are explained by the decrease of Cd content in $\mathrm{ZnCdSe}$ layer due to enhancement of $\mathrm{Cd} / \mathrm{Zn}$ interdiffusion process in the result of the increase of vacancy related defect number.
\end{abstract}

Keywords: Self-assembled quantum dots, II-VI semiconductors, Cd diffusion, photoluminescence.

Paper received 07.06.03; accepted for publication 17.06.03.

\section{Introduction}

Self-assembled quantum dot heterostructures based on II-VI semiconductors have attracted much attention in the past years as the convenient objects to study the fundamental aspects of self-assembly phenomenon as well as the promising candidates for fabrication of visible light-emitting devices. The most intensively studied system so far is CdSe on $\mathrm{ZnSe}$. The first blue-green $\mathrm{ZnSe}$ based room temperature optically pumped lasers and injection cw laser diodes containing layers with $\mathrm{ZnCdSe}$ quantum dots as active layers have been fabricated recently [1]. They demonstrated higher stability and lower threshold of excitation power density in comparison with similar devices based on quantum well structures.

The CdSe/ZnSe heteropair is very similar to well-studied InAs/GaAs one with respect to band gap difference $(\sim 1 \mathrm{eV})$ and lattice mismatch $(6.7 \%)$. However, unlike III-V system the process of CdSe/ZnSe nanoisland (quantum dot) formation is more complicated and is strongly influenced by $\mathrm{CdSe} / \mathrm{ZnSe}$ intermixing and $\mathrm{Cd}$ segrega- tion [2]. $\mathrm{Cd} / \mathrm{Zn}$ interdiffusion hinders nanoisland formation while $\mathrm{Cd}$ segregation helps in this process. As a result a classical Stranski-Krastanow type islanding is hindered and $\mathrm{ZnCdSe}$ alloy-like quantum well with CdSeenriched flat islands slightly overhanging or floating within is formed. Thus, for $\mathrm{CdSe} / \mathrm{ZnSe}$ heterostructures it is rather difficult to provide suitable growth conditions under which highly organised, coherently strained nanoislands can be obtained.

Besides, in II-VI compounds the deviation from stoichiometry is a typical phenomenon. We have found earlier that in CdSe/ZnSe quantum dot heterostructures a lot of cation vacancy related defects can be present [3]. The experiments on thermal annealing of $\mathrm{CdSe} / \mathrm{ZnSe}$ quantum well heterostructures have shown that the $\mathrm{CdSe} / \mathrm{ZnSe}$ intermixing significantly enhances in the presence of cation vacancies since $\mathrm{Cd}$ diffusion seems to proceed via the cation lattice points [4]. One can expect that cation vacancy related defects can influence also the processes of $\mathrm{ZnCdSe}$ nanoisland formation. So, in the present work a spatial distribution of cation vacancy related defects 


\section{L.V. Borkovska et al.: Influence of cation vacancy related defects ...}

and their influence on the optical characteristics and nanoisland formation processes in $\mathrm{CdSe} / \mathrm{ZnSe}$ heterostructures were investigated.

\section{Experimental procedure}

The structures studied were grown on GaAs (001) substrates by molecular beam epitaxy (MBE) and contained 12 vertically stacked CdSe inserts separated by ZnSe layers of thickness about $15 \mathrm{~nm}$. Nominal thickness of CdSe inserts varied from 2 up to 3.5 monolayers (ML). All samples were grown on a $200 \mathrm{~nm}$ thick ZnSe buffer layer and capped by a $100 \mathrm{~nm}$ thick ZnSe layer. $\mathrm{ZnSe}$ buffer and $\mathrm{ZnSe}$ barriers were grown at the temperatures of $280^{\circ} \mathrm{C}$ and $230{ }^{\circ} \mathrm{C}$, respectively. To stimulate the process of three-dimensional island formation after the deposition of each CdSe layer the $\mathrm{Cd}$ beam was blocked and the structure was heated up to $340{ }^{\circ} \mathrm{C}$ and then cooled down to $230^{\circ} \mathrm{C}$ under Se flux. Time duration of both the increase and the decrease in temperature was $4 \mathrm{~min}$. The reflection high energy electron diffraction was used for in situ control of the transition from twodimensional to three-dimensional growth mode. To increase a number of cation vacancy related defect a VI/II group beam pressure ratio $\mathrm{P}_{\mathrm{VI}} / \mathrm{P}_{\mathrm{II}}$ was changed from $2: 1$ to 5:1 (low and high Se beam pressure, respectively)..

Photoluminescence (PL) and photoluminescence excitation spectra were measured at $77 \mathrm{~K}$. Photoluminescence was excited by $337 \mathrm{~nm}$ line of $\mathrm{N}_{2}$-laser. For PLE measurements a light of xenon lamp dispersed by grating monochromator was used.

\section{Experimental results}

In the PL spectra of the heterostructures studied an intense emission band $I_{\mathrm{QD}}$ with maximum in blue-green spectral region is observed. We have found earlier [3] that optical characteristics of this band is in agreement with the results obtained for the structures grown by the similar methods [5] where such band was interpreted as radiative recombination of ground state heavy-hole-like exciton in $\mathrm{ZnCdSe}$ nanoislands. Fig. 1 shows the dependence of peak position and shape of this band on the nominal thickness of CdSe insert in the samples grown under low Se beam pressure. It is seen that the increase of the nominal thickness of CdSe insert results in consecutive red shift of $I_{\mathrm{QD}}$ band position. It is usually ascribed to the increase of Cd content in $\mathrm{ZnCdSe}$ nanoislands [2]. However, the halfwidth of $I_{\mathrm{QD}}$ band nonmonotonically depends on the thickness of CdSe inserts: at first it increases with the increase of CdSe thickness and after $3 \mathrm{ML}$ decreases. The largest value of $I_{\mathrm{QD}}$ band halfwidth $(\sim 130 \mathrm{meV})$ is observed in the samples with CdSe inserts of the nominal thickness of 2.5-3 ML. It is explained by the distinct two-component nature of this band.

Fig.2 shows the PL spectra of CdSe/ZnSe quantum dot heterostructures containing CdSe layers of the nominal thickness of $3 \mathrm{ML}$ grown under VI/II beam pressure

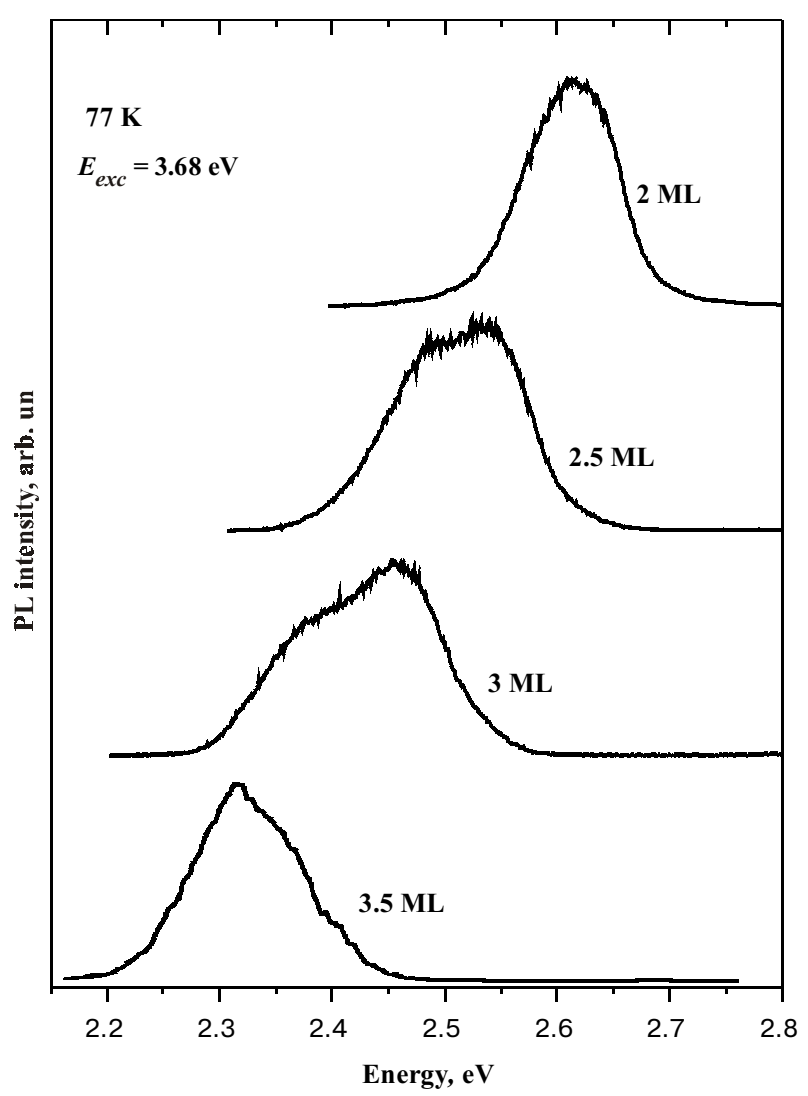

Fig. 1. PL spectra of $\mathrm{CdSe} / \mathrm{ZnSe}$ quantum dot heterostructures with $2 \div 3.5$ ML CdSe inserts grown under $\mathrm{P}_{\mathrm{VI}} / \mathrm{P}_{\mathrm{II}}=2: 1$.

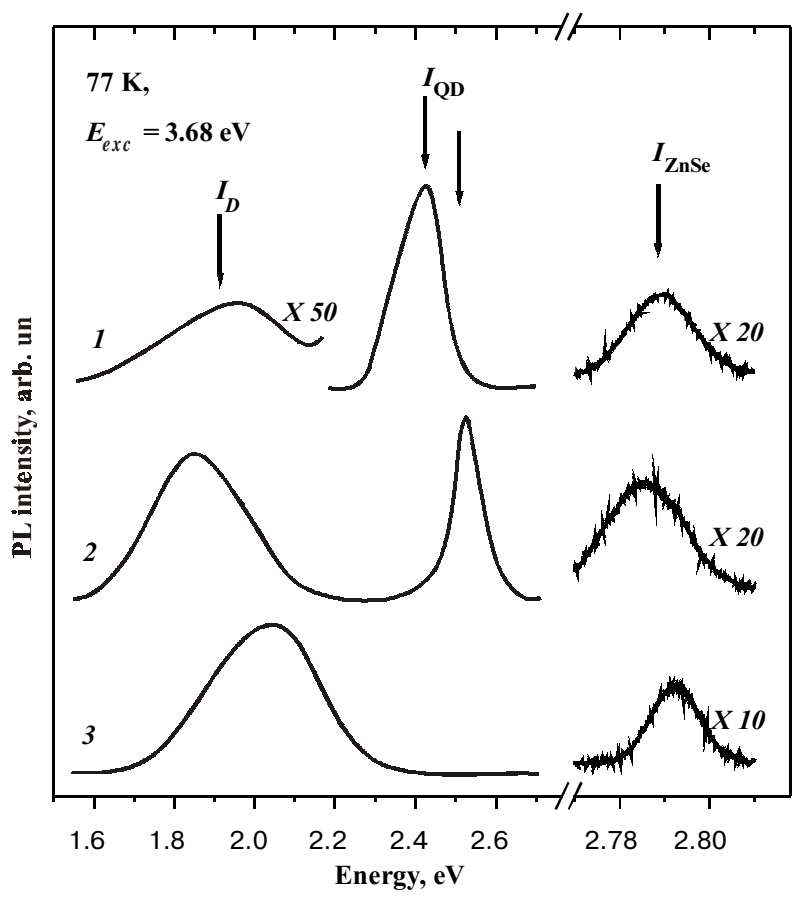

Fig. 2. PL spectra of $\mathrm{ZnSe}$ epilayer (curve 3) and $\mathrm{CdSe} / \mathrm{ZnSe}$ quantum dot heterostructures (curves 1,2 ) with $3 \mathrm{ML} \mathrm{CdSe}$ inserts grown under $\mathrm{P}_{\mathrm{VI}} / \mathrm{P}_{\mathrm{II}}=2: 1$ (curve 1 ) and 5:1 (curve 2). 


\section{L.V. Borkovska et al.: Influence of cation vacancy related defects ...}

ratio of 2:1 (curve 1) and 5:1 (curve 2). For comparison the PL spectrum of $0.6 \mathrm{~mm} \mathrm{ZnSe} \mathrm{epilayer} \mathrm{is} \mathrm{presented} \mathrm{on}$ Fig. 2 by curve 3 .

It is straightforward to see that the increase of Se beam pressure results in the narrowing of the $I_{\mathrm{QD}}$ band and shift of its spectral position to high energy side. In addition an excitation power density dependence of $I_{\mathrm{QD}}$ band intensity also changes. At low excitation level the intensity of $I_{\mathrm{QD}}$ band was several times smaller in the samples grown under $\mathrm{P}_{\mathrm{VI}} / \mathrm{P}_{\mathrm{II}}=5: 1$ than in the samples grown under $\mathrm{P}_{\mathrm{VI}} / \mathrm{P}_{\mathrm{II}}=2: 1$. However, at high excitation level the intensity of $I_{\mathrm{QD}}$ band became comparable in both groups of the samples (Fig. 2, curves 1,2).

In addition to $I_{\mathrm{QD}}$ emission two other luminescence bands are observed in the PL spectra of the $\mathrm{CdSe} / \mathrm{ZnSe}$ quantum dot heterostructures: the narrow peak $I_{\mathrm{ZnSe}}$ with the maximum at $2.79 \mathrm{eV}$ and the broad band $I_{\mathrm{D}}$ at 1.8 $1.95 \mathrm{eV}$ (Fig. 2). These two bands are also observed in the PL spectrum of the ZnSe epilayer (Fig. 2, curve 3). As our previous investigations showed [6] the first peak corresponds to $\mathrm{ZnSe}$ interband transition while the second one is caused by radiative transition of electron from conduction band to an acceptor level of defect including $\mathrm{Zn}$ vacancy $[6,3]$.

It is seen that the increase of VI/II beam pressure ratio results in the increase of $I_{\mathrm{D}}$ band intensity more that an order of value that can be attributed to the increase of a number of cation vacancy related defects. In addition, the position of $I_{\mathrm{D}}$ band maximum in the samples grown under $\mathrm{P}_{\mathrm{VI}} / \mathrm{P}_{\mathrm{II}}=5: 1$ is shifted to the low energy side while in the samples grown under low Se beam pressure it is close to the position of $I_{\mathrm{D}}$ band in $\mathrm{ZnSe}$ epilayer $(2.04 \mathrm{eV})$.

The spectral position of $I_{\mathrm{ZnSe}}$ band in the $\mathrm{CdSe} / \mathrm{ZnSe}$ heterostructures (Fig. 2, curves 1,2) is shifted to low energy side in comparison with $I_{\mathrm{ZnSe}}$ band position in $\mathrm{ZnSe}$ epilayer (Fig. 2, curve 3). This shift is the largest in the sample grown under high Se beam pressure (curve 2). In the samples grown under identical growth conditions the $I_{\mathrm{ZnSe}}$ band position shifts to low energy region with the increase of CdSe layer nominal thickness also.

To elucidate the spatial distribution of vacancy related defects and its possible influence on the luminescence characteristics of nanoislands the PL excitation spectra of both $I_{\mathrm{D}}$ and $I_{\mathrm{QD}}$ related bands were measured.

The excitation spectra of $I_{\mathrm{QD}}$ and $I_{\mathrm{D}}$ emission bands in the samples grown under low Se beam pressure and containing CdSe inserts of the nominal thickness of $2 \mathrm{ML}$ (Fig. 3, a) and 3 ML (Fig. 3, b) are presented on Fig.3 by curves 2 and 3 , respectively. In all curves the features connected with fundamental absorption edge of $\mathrm{ZnSe}$ layers $(\sim 2.8 \mathrm{eV})$ and light absorption in $\mathrm{ZnCdSe}$ wetting layer are observed. In addition, in the excitation spectra of nanoisland related band of the sample containing 2 ML CdSe inserts a peak that practically coincides with $I_{\mathrm{QD}}$ band position appears. It is natural to ascribe this maximum to light absorption in nanoislands [8]. It is seen that the increase of the nominal thickness of CdSe insert results not only in the shift of $I_{\mathrm{QD}}$ band position to the low energy side but also to the low energy shift of $\mathrm{ZnCdSe}$
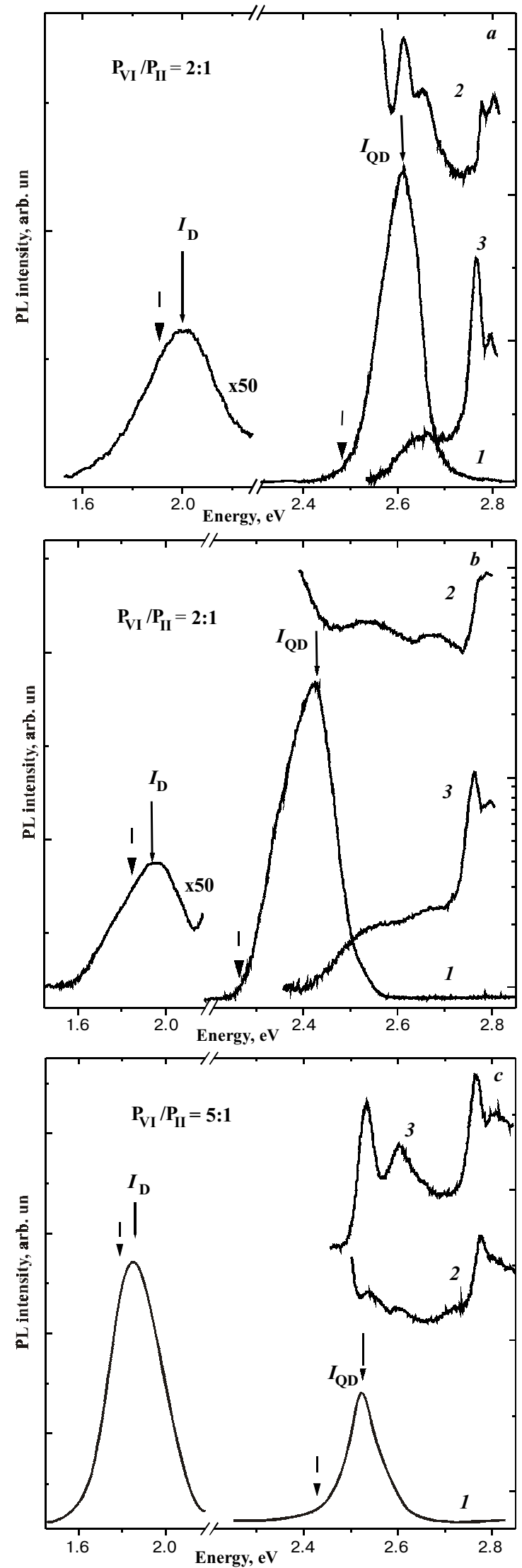

Fig. 3. PL spectra (curves 1 ), $I_{\mathrm{QD}}$ band (curves 2) and $I_{\mathrm{D}}$ band (curves 3) excitation spectra of $\mathrm{CdSe} / \mathrm{ZnSe}$ quantum dot structures with $2 \mathrm{ML}(a)$ and $3 \mathrm{ML}(b, c) \mathrm{CdSe}$ inserts grown under $\mathrm{P}_{\mathrm{VI}} / \mathrm{P}_{\mathrm{II}}=2: 1(a, b)$ and $5: 1(c) . T=77 \mathrm{~K}, P_{\text {exc }}=3.68 \mathrm{eV}$. The detection energies are marked by dashed arrows.

SQO, 6(3), 2003 


\section{L.V. Borkovska et al.: Influence of cation vacancy related defects ...}

wetting layer absorption edge (see curves 3 on Fig. 3, a, b). The latter can be explained by the increase of $\mathrm{Cd}$ content in $\mathrm{ZnCdSe}$ wetting layer [2]. In addition the energy distance between the $I_{\mathrm{QD}}$ band maximum position and absorption edge of the wetting layer also increases. This testifies to the rise of the depth of potential fluctuations in $\mathrm{ZnCdSe}$ layer with the increase of CdSe thickness.

Fig. 3, c shows the excitation spectra of $I_{\mathrm{QD}}$ and $I_{\mathrm{D}}$ emission bands in the $3 \mathrm{ML}$ sample grown under high $\mathrm{Se}$ beam pressure. One can see that here the peak caused by light absorption in nanoislands appears in the excitation spectra of both $I_{\mathrm{D}}$ and $I_{\mathrm{QD}}$ bands. It should be noted that the peak caused by light absorption in nanoislands is observed in the $I_{\mathrm{D}}$ band excitation spectra only in the samples grown under $\mathrm{P}_{\mathrm{VI}} / \mathrm{P}_{\mathrm{II}}=5: 1$.

\section{Discussion}

As it was mentioned in the previous section the increase of VI/II beam pressure ratio results in the increase of a number of cation vacancy related defects. However, the excitation spectra of $I_{\mathrm{D}}$ band show that spatial distribution of these defects changes also. In the excitation spectra of $I_{\mathrm{D}}$ band of all samples the features connected with light absorption in $\mathrm{ZnSe}$ layers and wetting $\mathrm{ZnCdSe}$ layers are observed. In the samples grown under high $\mathrm{Se}$ beam pressure the feature corresponded to light absorption in nanoislands is found in addition. It indicates that vacancy related defects generated in $\mathrm{ZnCdSe}$ layer are localised mainly in the wetting layer. But in the case of high number of these defects they are observed on nanoisland interface also.

It can explain a red shift of the $I_{\mathrm{D}}$ band maximum position in the samples grown under $\mathrm{P}_{\mathrm{VI}} / \mathrm{P}_{\mathrm{II}}=5: 1$. In fact, we have shown earlier [6] that the position of $I_{\mathrm{D}}$ band maximum depends on the relative contribution of defects located in different parts of heterostructure: $\mathrm{ZnSe}$ layers, $\mathrm{ZnCdSe}$ wetting layers and on nanoisland interface. In the last two cases the $I_{\mathrm{D}}$ band position is shifted to low energy side in comparison with one in $\mathrm{ZnSe}$ layer since the energy level of defect is associated with the quantized heavy-hole level of wetting layer or nanoislands [7]. As it follows from Fig. 3, b, c, the position of nanoisland related peak in the excitation spectrum of $I_{\mathrm{D}}$ band in the sample grown under $\mathrm{P}_{\mathrm{VI}} / \mathrm{P}_{\mathrm{II}}=5: 1$ nearly coincides with the wetting layer edge in the sample grown under $2: 1$. So, more low energy position of $I_{\mathrm{D}}$ band in the former case can be assigned to lower contribution of defects localised in $\mathrm{ZnSe}$ layers. It is proved by the relationship of the intensities of the features connected with light absorption in $\mathrm{ZnSe}$ layers and in wetting layer (Fig. 3, b) or nanoislands (Fig. 3, c).

It should be noted that the presence of vacancy related defects on nanoisland interface in the samples grown under high Se beam pressure influences on $I_{\mathrm{QD}}$ band intensity. They introduce an additional recombination channel that limits an efficiency of the nanoisland emis- sion under low excitation level. It is confirmed by the different dependence of the $I_{\mathrm{QD}}$ band intensity on excitation power density in the structures grown under low and high Se beam pressure.

The increase of VI/II beam pressure ratio results not only in the increase of a number and spatial distribution of cation vacancy related defects but also in nanoisland ensemble transformation.

Detailed transmission microscopy investigations [2, 9] have shown that CdSe insert transforms into relatively wide $\mathrm{ZnCdSe}$ wetting layer containing 2 types of nanoislands: the islands with lateral dimensions $<10 \mathrm{~nm}$ (nanoislands of type A) and the islands with lateral dimensions 15-30 nm (nanoislands of type B). In addition to higher dimensions the type $\mathrm{B}$ islands contain higher $\mathrm{Cd}$ concentration than type A ones. The nanoislands of type $\mathrm{A}$ with a density about $10^{11} \mathrm{~cm}^{-2}$ are formed even at submonolayer thickness of CdSe insert. They can be considered as fluctuations of quantum well. The nanoislands of type B appear when the critical thickness of CdSe layer on $\mathrm{ZnSe}$ (approximately $2 \mathrm{ML}$ ) is reached and are assigned to a Stranski-Krastanow growth process. Their density strongly depends on the CdSe coverage but does not exceed the density of type A islands.

As it follows from Fig. 3, our samples grown under low Se beam pressure also contain two types of nanoislands. Exciton recombination in type A nanoislands dominates in the PL spectra of the sample with $2 \mathrm{ML}$ $\mathrm{CdSe}$ inserts while emission from type B islands prevails in the case of $3 \mathrm{ML}$ inserts. It becomes apparent from the excitation spectra of $I_{\mathrm{QD}}$ band of these two samples. In the case of type A islands the excitation spectrum of $I_{\mathrm{QD}}$ band contains the peak caused by optical absorption in nanoislands (Fig. 1,a). It means that these nanoislands can interact via tunneling or phonon assisted hopping like quantum well fluctuations [5]. In the case of type B islands these processes are strongly suppressed and no nanoisland related peak is observed in the $I_{\mathrm{QD}}$ band excitation spectrum (Fig. 3,b). Additional evidence of type $\mathrm{B}$ nanoisland formation in the samples with $3 \mathrm{ML} \mathrm{CdSe}$ inserts is the distinct two-component shape of $I_{\mathrm{QD}}$ band (Fig. 1).

However, in the samples grown under high Se beam pressure even at $3 \mathrm{ML}$ thickness of CdSe inserts the nanoislands of type A arise predominantly (Fig. 3, c). It is confirmed by the presence of the feature caused by the light absorption in nanoislands in the excitation spectrum of $I_{\mathrm{QD}}$ band as well as smaller halfwidth of $I_{\mathrm{QD}}$ band.

As a rule more high energy position of nanoisand emission band is connected with smaller Cd content in nanoislands [2]. Thus, we can suppose that observed change of nanoisland emission band with the increase of Se beam pressure is caused by the decrease of $\mathrm{Cd}$ content in nanoislands and suppression of type B nanoisland formation.

It was found [10] that the increase of VI/II group beam pressure ratio hampers the formation of MBE-grown $\mathrm{ZnCdSe}$ nanoislands. It was supposed to be due to sup- 


\section{L.V. Borkovska et al.: Influence of cation vacancy related defects ...}

pression of segregation process as it was observed in III-V compounds [11]. However, $\mathrm{Cd} / \mathrm{Zn}$ interdiffusion can produce the same effect. Significant enhancement of interdiffusion processes can be expected in the case of high concentration of cation vacancies [4]. Our investigations show that high number of cation vacancies is generated in the case of high Se beam pressure. Thus, we can expect essential enhancement of interdiffusion processes in the structures grown under high Se beam pressure.

On the other hand, interdiffusion efficiency has to depend on localization of cation vacancies. Presence of cation vacancies in wetting layer as well as on nanoisland interface can stimulate not only the intermixing of the components of $\mathrm{ZnCdSe}$ inserts and $\mathrm{ZnSe}$ barriers but also in smoothing of potential fluctuation $\mathrm{ZnCdSe}$ layers.

$\mathrm{Cd}$ diffusion in $\mathrm{ZnSe}$ layers has to manifest itself in the change of their structural and optical characteristics. It should result in formation of $\mathrm{Cd}$ enriched regions in $\mathrm{ZnSe}$ layers adjacent to $\mathrm{ZnCdSe}$ insert. Such $\mathrm{Cd}$ enriched regions can be either $\mathrm{ZnSe}$ layers doped with cadmium isovalent impurity or low concentration $\mathrm{ZnCdSe}$ solid solution. Their formation has to develop in the low energy shift and widening of $\mathrm{ZnSe}$ band-to-band emission.

We indeed observed such changes of this band. We have found that the increase of the nominal thickness of CdSe insert results in low energy shift of $I_{\mathrm{ZnSe}}$ band and its halfwidth increase. It can be assigned to interdiffusion process because: (i) the increase of CdSe nominal thickness should result in the increase of $\mathrm{Cd}$ concentration in the insert [2]; (ii) more intense penetration of $\mathrm{Cd}$ in $\mathrm{ZnSe}$ layers can be expected due to greater concentration gradient. The increase of Se beam pressure also results in low energy shift of $I_{\mathrm{ZnSe}}$ band and its halfwidth increase. It testifies to the enhancement of diffusion process in the samples containing high number of cation vacancy related defects.

Thus, observed changes in optical and structural characteristics of $\mathrm{ZnSe}$ layers indicates the enhancement of interdiffusion process with the increase of Se beam pressure. Decrease of Cd concentration in nanoislands due to interdiffusion can explain an observed high energy shift of $I_{\mathrm{QD}}$ band.

\section{Conclusions}

In the present paper intrinsic defects and their influence on optical characteristics of $\mathrm{CdSe} / \mathrm{ZnSe}$ quantum dot heterostructures were studied by photoluminescence and photoluminescence excitation methods. In all the samples the self activated emission connected with donoracceptor pairs $\mathrm{V}_{\mathrm{Zn}}$-D and caused by radiative capture of electron from conduction band by acceptor level was observed. Analysis of the excitation spectra of this band revealed that vacancy related defects are present in $\mathrm{ZnCdSe}$ wetting layer. The increase of VI/II beam pressure ratio from $2: 1$ to $5: 1$ results in the increase of $I_{\mathrm{D}}$ band intensity more that an order of value that can be attributed to the increase of a number of cation vacancy related defects.. In this case these defects are observed on nanoisland interface and introduce an additional recombination channel that limits an efficiency of the nanoisland emission under low excitation level. The increase of VI/II beam pressure results also in significant change of other emission bands in the photoluminescence spectra: (i) high energy shift and narrowing of nanoisland emission band, (ii) low energy shift and widening of $\mathrm{ZnSe}$ band-to-band emission peak. We attribute this changes to enhancement of $\mathrm{Cd} / \mathrm{Zn}$ interdiffusion process due to increase of cation vacancy defect number. It allows us to conclude that hampering of nanoisland formation with the increase of VI/II beam pressure ratio is caused by enhancement of interdiffusion process.

\section{References}

1. S.V. Ivanov, A.A. Toropov, S.V. Sorokin, T.V. Shubina, A.V. Lebedev, I.V. Sedova, A.A. Sitnikova, R.V. Zolotareva, P.S. Kop'ev, Zh.I. Alferov, Ultra-low threshold ZnSe-based lasers with novel design of active region // J. Cryst. Growth 201/202, pp. 942-945 (1999).

2. N. Peranio, A. Rosenauer, D. Gerthsen, S.V. Sorokin, I.V. Sedova, S.V. Ivanov, Structural and chemical analysis of $\mathrm{CdSe} / \mathrm{ZnSe}$ nanostructures by transmission electron microscopy // Phys. Rev. B, 61(23), pp. 16015-16024 (2000).

3. G.N. Semenova, Ye.F. Venger, M.Ya. Valakh, Yu.G. Sadofyev, N.O. Korsunska, V.V. Strelchuk, L.V. Borkovska, V.P. Papusha, M.V. Vuychik, Optical investigations of the influence of point defects on quantum dots in $\mathrm{CdSe} / \mathrm{ZnSe}$ heterostructures // $\mathrm{J}$. Phys.: Condens. Matter, 14, pp. 13375-13380 (2002).

4. M. Kuttler, M. Strassburg, V. Turck, R. Heitz, U.W. Pohl, D. Bimberg, E. Kutz, G. Landwehr, D. Hommel, // Appl. Phys. Lett., 69, pp. $2647-2649$ (1996).

5. E. Kurtz, J. Shen, M. Schmidt, M. Grun, S.K. Hong, D. Litvinov, D. Gerthsen, T. Oka, T. Yao, C. Klingshirn, Formation and properties of self-organised II-VI quantum islands // Thin Sol. Films 367, pp. 68-74 (2000).

6. G.N. Semenova, E.F. Venger, N.O. Korsunska, V.P. Klad'ko, L.V. Borkovska, M.P. Semtsiv, M.B. Sharibaev, V.I. Kushnirenko, Yu.G. Sadofyev, Defects and radiation-enhanced defect reactions in $\mathrm{ZnSe} /(001) \mathrm{GaAs} \mathrm{MBE}$ layers // $S Q O, \mathbf{5}(2)$, pp. 133-137 (2002).

7. M.Ya. Valakh, Yu.G. Sadofyev, N.O. Korsunska, G.N. Semenova, V.V. Strelchuk, L.V. Borkovska, M.V. Vuychik, M. Sharibaev, Deep-level defects in $\mathrm{CdSe} / \mathrm{ZnSe}$ QDs and giant anti-Stokes photoluminescence // SQO, 5(3) pp. 254-257 (2002).

8. I.L. Krestnikov, M. Strasburg, M. Caesar, A. Hoffmann, U.W. Pohl, D. Bimberg, N.N. Ledentsov, P.S. Kop'ev, Zh.I. Alferov, D. Litvinov, A.Rosenauer, D. Gerthsen, Control of the electronic properties of CdSe submonolayer superlattices via vertical correlation of quantum dots // Phys. Rev. B., 60(12), pp. 8695-8703 (1998).

9. D. Schikora, S. Schwedhelm, D.J. As, K. Lischka, D. Litvinov, A. Rosenauer, D. Gerthsen, M. Strassburg, A. Hoffmann, D. Bimberg, Investigations on the Stranski-Krastanow growth of CdSe quantum dots // Appl. Phys. Lett., 76(4) pp. 418-420 (2000).

10. T. Passow, K. Leonardi, H. Heinke, D. Hommel, J. Seufert, G. Bacher, A. Forchel,Influence of capping conditions on $\mathrm{CdSe} / \mathrm{ZnSe}$ quantum dot formation // Phys. Stat. Sol. (b) 229(1), pp. 497-501 (2002).

11. K. Muraki, S. Fukatsu, Y. Shiraki, R. Ito, Surface segregation of In atoms during molecular beam epitaxy and its influence on the energy levels in InGaAs/GaAs wells // Appl. Phys. Lett., 61(5), pp. 557-559 (1992). 